\title{
Pacemakers in MRI for the Neuroradiologist
}

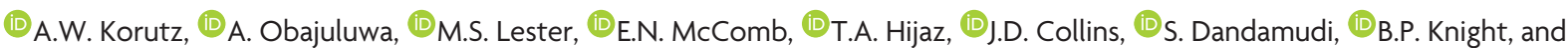

(1)A.J. Nemeth

\begin{abstract}
SUMMARY: Cardiac implantable electronic devices are frequently encountered in clinical practice in patients being screened for MR imaging examinations. Traditionally, the presence of these devices has been considered a contraindication to undergoing MR imaging. Growing evidence suggests that most of these patients can safely undergo an MR imaging examination if certain conditions are met. This document will review the relevant cardiac implantable electronic devices encountered in practice today, the background physics/ technical factors related to scanning these devices, the multidisciplinary screening protocol used at our institution for scanning patients with implantable cardiac devices, and our experience in safely performing these examinations since 2010.
\end{abstract}

ABBREVIATIONS: CIED = cardiac implantable electronic device; EP = electrophysiologist; ICD = implantable cardioverter-defibrillator; $\mathrm{RF}=$ radiofrequency; SAR = specific absorption rate

$\mathrm{M}_{\mathrm{i}}^{\mathrm{R}}$ $\mathrm{R}$ imaging examinations are being performed with increasing frequency worldwide, with nearly 35 million scans performed annually in the United States alone. ${ }^{1}$ More than 1.8 million individuals in the United States have a cardiac pacemaker or implantable cardioverter-defibrillator (ICD), both of which fall under the larger umbrella term of cardiac implantable electronic devices (CIEDs). Traditionally, these devices have precluded patients from undergoing MR imaging examinations. ${ }^{2-5}$ More recently, "MR imaging-conditional" CIEDs have been developed, which contain components that can be safely placed in an MR imaging scanner. ${ }^{6}$ Despite the development of these devices, many patients with a CIED that is not MR imaging-conditional remain and would benefit clinically from an MR imaging examination.

To date, a growing body of research suggests that CIEDs that were traditionally considered MR imaging incompatible can be scanned safely when certain precautions are taken. ${ }^{2,7-10}$ Despite these published reports, many institutions still do not have protocols in place to scan patients with CIEDs.

In this article, we will begin by providing an overview of the types of CIEDs encountered in practice today as well as some of the relevant physics and technical factors related to scanning these devices. Then we will discuss the multidisciplinary protocol used

From the Departments of Radiology (A.W.K., A.O., M.S.L., E.N.M., T.A.H., J.D.C., A.J.N.), Medicine, Division of Cardiology (S.D., B.P.K.), and Neurology (A.J.N.), Northwestern University Feinberg School of Medicine, Chicago, Illinois.

Please address correspondence to Alexander W. Korutz, MD, 676 N St Clair St, Suite 800, Chicago, IL 60611; e-mail: Alexander.Korutz@nm.org

- Indicates open access to non-subscribers at www.ajnr.org

http://dx.doi.org/10.3174/ajnr.A5314 at our institution for scanning patients with CIEDs, including initial cardiac evaluation/cardiac clearance, cardiac monitoring during scanning, and the relevant postscan evaluation and clinical follow-up. Finally, we will outline the number and types of procedures performed at our institution since 2010 and any complications encountered during this period.

\section{Cardiac Implantable Electronic Devices}

A pacemaker is an implantable device that senses cardiac activity and delivers the required electrical stimuli to the heart to regulate slow heart rates or erratic cardiac rhythms. An ICD is a device that contains both pacemaker and defibrillator components, the latter of which can provide a high-energy shock for treating life-threatening arrhythmias, most commonly ventricular tachyarrhythmias. These devices are currently classified under the more general term "cardiac implantable electronic devices," and they consist of a pulse generator and leads that extend into $\geq 1$ of the chambers of the heart. The pulse generator contains the relevant circuitry for the device and the device battery. The CIED leads are bidirectional wires that function to sense intrinsic cardiac activity and deliver electrical impulses to the heart. ${ }^{11-13}$

As described above, a CIED may have $\geq 1$ cardiac lead. If only a single lead is present, it is usually implanted into either the right ventricle or right atrium. When a 2-lead CIED is present, the leads are typically implanted into both the right atrium and right ventricle, with the latter lead performing both pacemaker and defibrillator functions. Biventricular pacing, also called "cardiac resynchronization therapy," uses a third lead, which is usually implanted in a ventricular branch of the coronary sinus to capture 
Table 1: Pacing modes

\begin{tabular}{lll}
\multicolumn{1}{c}{$\begin{array}{c}\text { Chamber } \\
\text { Paced }\end{array}$} & \multicolumn{1}{c}{$\begin{array}{c}\text { Chamber } \\
\text { Sensed }\end{array}$} & $\begin{array}{c}\text { Pacer Response to } \\
\text { Sensed Beat }\end{array}$ \\
\hline $\mathrm{V}=$ ventricle & $\mathrm{V}=$ ventricle & $\mathrm{I}=$ inhibited \\
$\mathrm{A}=$ atrium & $\mathrm{A}=$ atrium & $\mathrm{T}=$ triggered \\
$\mathrm{D}=$ both (dual) & $\mathrm{D}=$ both (dual) & $\mathrm{D}=$ inhibited or triggered \\
& & $\begin{array}{c}\text { depending on chamber } \\
\text { (dual) }\end{array}$ \\
$\mathrm{O}=$ none & $\mathrm{O}=$ none & $\mathrm{O}=$ none \\
\hline
\end{tabular}

the left ventricular epicardium at the same time as right ventricular activation to improve synchronization between right and left ventricular contractions. ${ }^{1-13}$ Regardless of whether the patient has a pacemaker device or a combined pacemaker/defibrillator and irrespective of the number of device leads, a CIED is programmed to operate in a specific pacing mode chosen to provide the greatest benefit to the patient.

In the simplest terms, the pacing mode is denoted with a 3-letter code (eg, DDD or VOO), with both the letter position and letter type describing specific pacemaker functions. Fourth and fifth letters can be added to this code; however, discussion of these is beyond the scope of this article. The first letter describes which chamber is being paced, the second describes which chamber is being sensed, and the third describes how the pacemaker responds when a beat is sensed (Table 1). While the first 2 letters in this code are self-explanatory, the third letter requires a brief discussion. During "inhibition" mode, a pacemaker will inhibit ventricular pacing when a heartbeat is sensed. "Trigger" mode indicates that the device will trigger a ventricular pacing stimulus every time an atrial beat is sensed. Finally, "dual" mode indicates a more complex situation in which the device responds to a sensed beat in the atrium or ventricle by inhibiting pacing output to that chamber and simultaneously delivering a stimulus to the ventricle after the atrial beat is sensed. This scenario only arises if there is no inhibition of the pacemaker by an intrinsic beat originating in the ventricle. For example, when a patient's device is programmed in DDD mode, the device may operate in 4 possible ways: pace both chambers, sense only in both chambers without pacing, pace the atrium with natural conduction to the ventricle, or pace the ventricle following an intrinsic atrial beat. Finally, asynchronous (also known as "fixed") pacing can be used as a more general term to describe any scenario in which cardiac pacing is not inhibited by intrinsic cardiac activity. ${ }^{13}$ For instance, in VOO pacing, the ventricle is paced at a fixed rate with no device sensing, so there is no capacity to inhibit/trigger based on sensed activity.

\section{Current Experience of CIEDs with MR Imaging}

Traditional CIEDs are currently referred to as non-MR imagingconditional devices, given the advent of newer, MR imaging-conditional devices. The presence of a non-MR imaging-conditional CIED was conventionally thought to be a contraindication for undergoing MR imaging. ${ }^{14-16}$ As will be discussed shortly, this is no longer the case. Multiple risks have been associated with these nonconditional devices, primarily related to interactions between the ferromagnetic components of these devices and the static magnetic field, gradient magnetic fields, and the transmitted radiofrequency (RF) field. ${ }^{17}$
The static magnetic field can affect a CIED in multiple ways. For instance, the field can reset/reprogram the device or deplete its battery. ${ }^{4,18-20}$ It can also affect the magnetically activated reed switch within the device, spontaneously switching the CIED into an asynchronous pacing mode. ${ }^{21,22}$ The potential for some of these scenarios to occur can be limited by switching the device into asynchronous mode before placing the patient in the scanner and switching off the ability of an ICD to provide a therapeutic shock..$^{4,21,23}$ The static magnetic field can also interact with charged ions in moving blood, producing small local voltages that, when superimposed on the patient's electrocardiogram, can falsely mimic arrhythmias or other electrocardiogram changes. In some instances, this phenomenon may inhibit pacemaker function or falsely simulate the presence of a cardiac arrhythmia, which requires administration of a shock. ${ }^{4,21}$ Last, the static field can cause a mechanical torque on the device, which can move the pulse generator within the chest wall or dislodge the device leads within the myocardium..$^{21,24,25}$

Complications associated with the rapidly alternating gradient magnetic fields mainly involve the induction of currents within the device leads themselves. Similar to local currents produced by moving blood within the static field, currents within the cardiac leads can also mimic cardiac electrical activity, thereby inhibiting the need for pacing, pacing the heart at inappropriately high rates, or administering electronic shocks, depending on the scenario in which the above occurs. ${ }^{21,24,25}$ Alternatively, induced current at the lead tip could surpass the activation threshold needed to stimulate cardiac myocytes, thereby having the potential to generate life-threatening arrhythmias such as ventricular fibrillation and ventricular tachycardia. ${ }^{21,24}$

Finally, the RF pulse used during an MR imaging examination can also induce currents within device leads. Due to the high frequency of these RF pulses and the high conductivity of the device leads relative to the adjacent soft tissues, energy can be lost in the form of heat or so-called ohmic (also known as "resistive") heating. This heating is concentrated at the tip of a device lead or at a point where a lead is fractured. Resultant focal heating may cause adjacent tissue damage and, subsequently, the need for a higher pacing threshold or loss of pacing capture entirely. ${ }^{4,7,15,24,26,27}$

Despite these hypothetic risks when scanning a patient with a non-MR imaging-conditional CIED, recent statements from the American Heart Association and the European Society of Cardiology have suggested that MR imaging may be performed at a $1.5 \mathrm{~T}$ field strength when using specific protocols for programming and monitoring. ${ }^{4,5}$ These statements are based on results from a growing number of trials that have investigated this issue, focusing specifically on the potential risks and overall safety of MR imaging in patients with non-MR imaging-conditional devices. $^{2,7-10}$ For instance, Martin et $\mathrm{al}^{9}$ examined 54 non-pacerdependent patients with non-MR imaging-conditional pacemaker devices who underwent $62 \mathrm{MR}$ imaging examinations that included both thoracic and nonthoracic studies. This study found a change in the pacing threshold in $37 \%$ of device leads, of which most threshold changes were judged to be unimportant and no threshold changes were noted to have any clinical impact. ${ }^{9}$

In 2011, Nazarian et $\mathrm{al}^{2}$ reported their prospective experience following 438 patients with pacemakers and ICDs who underwent $555 \mathrm{MR}$ imaging examinations. This study included thoracic ex- 
aminations but excluded pacer-dependent patients with ICDs. The authors reported power-on resets in 3 of 438 patients, none of which were associated with long-term device dysfunction. With regard to lead parameters such as sensing, impedance, and capture thresholds, no device in this study required device revision or reprogramming due to any parameter changes. However, right ventricular lead-sensed amplitude was lower and right ventricular capture thresholds tended to be higher both immediately following MR imaging examinations and at long-term device followup. These lead parameter changes were most strongly associated with thoracic MR imaging examinations, though they were not clinically important. ${ }^{2}$ Dandamudi et $\mathrm{al}^{10}$ evaluated 58 patients with non-MR imaging-conditional CIEDs who underwent only cardiac and thoracic spine MR imaging examinations. No clinically important parameter changes, arrhythmias, device mode changes/electrical resets, or battery depletions were found following the MR imaging examinations in this study.

More recently, the largest study to date, The MagnaSafe Registry (http://magnasafe.org/), examined 1500 nonthoracic MR imaging scans in patients with non-MR imaging-conditional CIEDs. ${ }^{8}$ In this study, device interrogation following the MR imaging examinations assessed parameter changes such as a decrease in battery voltage, an increase in pacing threshold, pacing or shock lead impedance change, and a decrease in P-and R-wave amplitudes. This study found that no patient who was appropriately screened and reprogrammed following the procedure had device or lead failure. The authors also noted that changes in device settings were uncommon and not clinically important. Of note, 1 case of the 1500 (in the subgroup of 500 patients with ICDs) did require device replacement due to device failure from inappropriate programming during the MR imaging examination. Additionally, there were 6 cases of partial electrical reset, which was corrected following the MR imaging examination during standard device reprogramming. Finally, 6 patients developed atrial fibrillation/flutter, though 5 of these patients had a history of paroxysmal atrial fibrillation and the sixth patient had resolution by 48 hours.

To date, an overwhelming body of evidence suggests that patients with intact CIEDs can be scanned safely when using proper MR imaging protocols and if the risk to the patient is well-managed. A few absolute contraindications remain for performing MR imaging in a patient with a CIED. The first is a device that was implanted $<6$ weeks before the MR imaging examination. These leads have not fully healed and are susceptible to dislodgement. ${ }^{2}$ The second is the presence of an abandoned or fractured lead. When the lead is not connected to a CIED generator, then, there is no heat sink in place for the lead. The third is the presence of surgically placed permanent epicardial pacing leads. The lead design for these devices is different from that in the more common transvenous leads, and there are insufficient data to recommend MR imaging in these patients at this time. ${ }^{4}$

A few additional pacing devices can be encountered in clinical practice, the 2 most common of which are temporary epicardial pacing devices and temporary intracardiac pacing devices. Both devices comprise an external pulse generator paired with $\geq 1$ cardiac lead. In all instances, the patient should not undergo an MR imaging examination when the external pulse generator is present, regardless of its type. External pulse generators for temporary pacing devices use less sophisticated designs, which make them more prone to electromagnetic interference. In addition, no studies of the safety of these devices in an MR imaging environment have been performed, to our knowledge. Retained (also known as "abandoned") temporary epicardial pacing leads are not believed to present a risk of injury during an MR imaging examination, given that these leads are relatively short and usually do not form large "antennalike" loops. Nevertheless, there is still a theoretic risk with these devices of cardiac excitation and thermal injury, though at our institution, this risk is not considered high enough to prevent scanning these patients. On the other hand, retained leads for temporary intracardiac (ie, transvenous) pacing devices are considered a contraindication for undergoing an MR imaging examination. These devices tend to have unfixed leads, which are more susceptible to movement, and longer leads, which are more prone to current induction. ${ }^{4}$

Given the traditional thinking that the presence of a non-MR imaging-conditional device was a contraindication for performing an MR imaging examination, there was a time when an unmet need existed for a CIED that could be safely placed in an MR imaging environment. In 2008, the first MR imaging-conditional device was released in the European market with subsequent release of a similar pacemaker in the United States in $2011 .{ }^{24} \mathrm{Mul}-$ tiple design changes were used to produce CIEDs that were safe for an MR imaging environment. These include constructing the device from materials that are less magnetosensitive (ie, nickel, cobalt, or chromium) or entirely nonferromagnetic, using a solidstate Hall-effect sensor rather than the somewhat unpredictable reed switch, protecting/desensitizing the electronic circuits within the CIED from energy deposition during the MR imaging examination, modifying the construction of the device leads, and using MR imaging-specific programing algorithms (ie, a specific "MR imaging mode"). ${ }^{21,25}$

For a CIED in a specific patient to be correctly labeled as MR imaging-conditional, the entire device, including the pulse generator, pulse generator software, and cardiac leads, must all be constructed from MR imaging-conditional components produced by the same manufacturer. In addition, each manufacturer requires that other specific conditions be met. These are related to magnet field strength (1.5T magnet only), time when the CIED was implanted ( $>6$ weeks before the MR examination), body part to be scanned, specific absorption rate (SAR; maximum of 2 $\mathrm{W} / \mathrm{kg}$ ), and so forth. ${ }^{21,24,25}$ Please see Table 2 for a list of currently approved MR imaging-conditional CIEDs.

Despite the wide availability of MR imaging-conditional CIEDs, non-MR imaging-conditional devices are still implanted in clinical practice today. In certain instances, non-MR imagingconditional devices are implanted for cost reasons. Non-MR imaging-conditional generators also continue to be installed in patients who have non-MR imaging-conditional devices, in which the leads are intact and the device generator must be replaced due to battery depletion.

\section{MR Imaging Physics and Technical Considerations Relevant to Pacemakers}

When an object is placed in an MR imaging scanner, hydrogen atoms tend to align either with or against the static magnetic field, 
Table 2: Currently approved MRI-conditional CIEDs

\begin{tabular}{|c|c|c|}
\hline $\begin{array}{c}\text { Device } \\
\text { Manufacturer }\end{array}$ & Device Model & Device Type \\
\hline Boston Scientific $^{a}$ & Accolade & Pacemaker \\
\hline Boston Scientific & Essentio & Pacemaker \\
\hline Boston Scientific & Emblem & Subcutaneous ICD \\
\hline BIOTRONIK ${ }^{b}$ & Entovis & Pacemaker \\
\hline BIOTRONIK & Eluna & Pacemaker \\
\hline BIOTRONIK & Edora & Pacemaker \\
\hline BIOTRONIK & Iperia 7 DRT-T/VR-T & $I C D$ \\
\hline BIOTRONIK & Iperia $7 \mathrm{HF}-\mathrm{T}$ & CRT \\
\hline Medtronic $^{c}$ & Advisa MRI & Pacemaker \\
\hline Medtronic & Revo MRI & Pacemaker \\
\hline Medtronic & Visia AF MRI & $I C D$ \\
\hline Medtronic & Evera MRI & $I C D$ \\
\hline Medtronic & Claria MRI & CRT \\
\hline Medtronic & Amplia MRI & CRT \\
\hline Medtronic & Compia MRI & CRT \\
\hline St. Jude Medical ${ }^{d}$ & Assurity MRI & Pacemaker \\
\hline
\end{tabular}

Note:-CRT indicates cardiac resynchronization therapy.

a Natick, Massachusetts.

b Lake Oswego, Oregon.

Minneapolis, Minnesota.

${ }^{\mathrm{d}}$ Minnetonka, Minnesota.

$\mathrm{B}_{0}$. A rotating radiofrequency pulse can then be applied that contains 2 orthogonally oriented components, the magnetic field (Bfield or $\mathrm{B}_{1}$ ) and the electric field (E-field). The positive component of the B-field tilts the hydrogen atoms into the transverse plane, where the atoms rotate and produce a signal detected in the receiver coil. As described previously, this RF pulse deposits energy in the tissue and results in tissue heating. ${ }^{4,21,28,29}$ The term that has been traditionally used to describe the RF power that is absorbed per unit mass of an object is the "specific absorption rate." SAR is expressed in watts per kilogram and is related to the square of the main magnetic field, square of the flip angle, square of the patient radius, patient conductivity, and the RF duty cycle. $^{29}$ "Duty cycle" refers to the percentage of time that the RF pulse is "on" and can be shortened by lengthening the TR.

Because the SAR calculation relies on estimates of patient size and composition, each scanner manufacturer uses proprietary models for calculating this value. Therefore, SAR values calculated for a patient may vary among scanner vendors. ${ }^{30}$ Thus, the term $B_{1+R M S}$ has been proposed as a replacement for SAR. Briefly, $\mathrm{B}_{1+\mathrm{RMS}}$ refers to the time-averaged RF magnetic field, which is generated by the RF coil. This term is independent of patient factors and is solely determined by MR imaging scanner parameters, such as flip angle, RF pulse duration, echo-train length, choice of a gradient-echo rather than a spin-echo sequence, and so forth. ${ }^{30,31}$

Per the Faraday law, a changing magnetic field will produce a voltage in an exposed electrical conductor. The resulting current depends on the speed with which the magnetic field changes $(\mathrm{dB} /$ $\mathrm{dt}$ ), the conductivity of the object, and the cross-sectional area of the conducting loop. Therefore, both the transmitted RF magnetic field and the time-alternating gradient magnetic fields are capable of producing currents within conductors, albeit to different degrees. ${ }^{17,32}$ The RF fields used in MR imaging are high-frequency pulses capable of producing high-frequency oscillating currents $(64 \mathrm{MHz}$ at $1.5 \mathrm{~T}$ ), which can deposit relatively large amounts of energy in tissue. On the other hand, the alternating

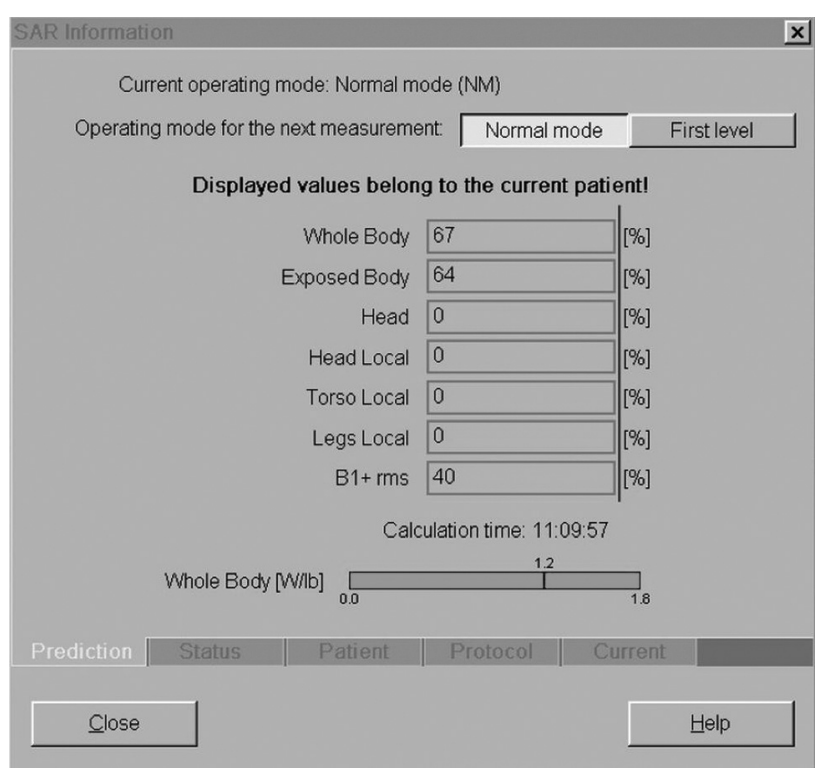

FIG 1. Normal versus first-level mode: normal level only for neuroradiology. SAR information panel at the scanner console for a sagittal STIR TSE acquisition of the lumbar spine. Any neurologic imaging study that can be run on a 1.5T magnet, including echo-planar imaging, can be performed in a patient with a CIED, as long as the examination is performed in normal operating mode. Image courtesy of Charles Fasanati.

gradient magnetic fields produce relatively low-frequency currents (ie, $<1000 \mathrm{~Hz}$ ), which deposit very low levels of energy in tissue. ${ }^{17,28,29}$ Therefore, a CIED generator and leads could serve as an antenna with which to generate and concentrate a current, a current concentrated at the lead tip or any site of lead fracture, if present. $4,15,23,26,27,32,33$

The quantity of energy deposited in a patient with a CIED also depends, in part, on the patient's location within the magnet and the configuration of the generator and device leads within the patient. In general, the greatest RF energy is deposited when the generator and leads are positioned entirely within the RF coil isocenter. ${ }^{7,9,26,32,34}$ In addition, the amount of energy deposited in the patient also increases as the length of the exposed device lead increases. The "exposed" lead refers to the length of wire that extends from the device generator to the insertion site in the myocardial tissue. A longer exposed lead creates a longer loop with which to interact with the RF field. ${ }^{32,33}$ Last, pacemaker generators placed within the right chest wall have also been shown to lead to greater tissue heating, even at times when a smaller lead area is present. This has been attributed to nonuniform RF generated E-fields within the scanner bore. ${ }^{32,34-37}$

While the focused examinations performed in clinical practice (ie, MR imaging of the brain) produce a local SAR value in the region being scanned, SAR is typically averaged over the entire body, generating a single whole-body SAR value. When performing an MR imaging examination, the operator (ie, technologist) can choose between scanning in normal and first-level modes. In normal mode, the whole-body SAR is limited to $2 \mathrm{~W} / \mathrm{kg}$. In firstlevel mode, there is an allowed maximum SAR of $4 \mathrm{~W} / \mathrm{kg}$. The scanning mode is selected at the MR imaging scanner console (Fig 1). $B_{1+R M S}$ values are also displayed in this region.

At our institution, all MR imaging in patients with pacemakers is performed at $1.5 \mathrm{~T}$, and the pacemaker must have been in place 


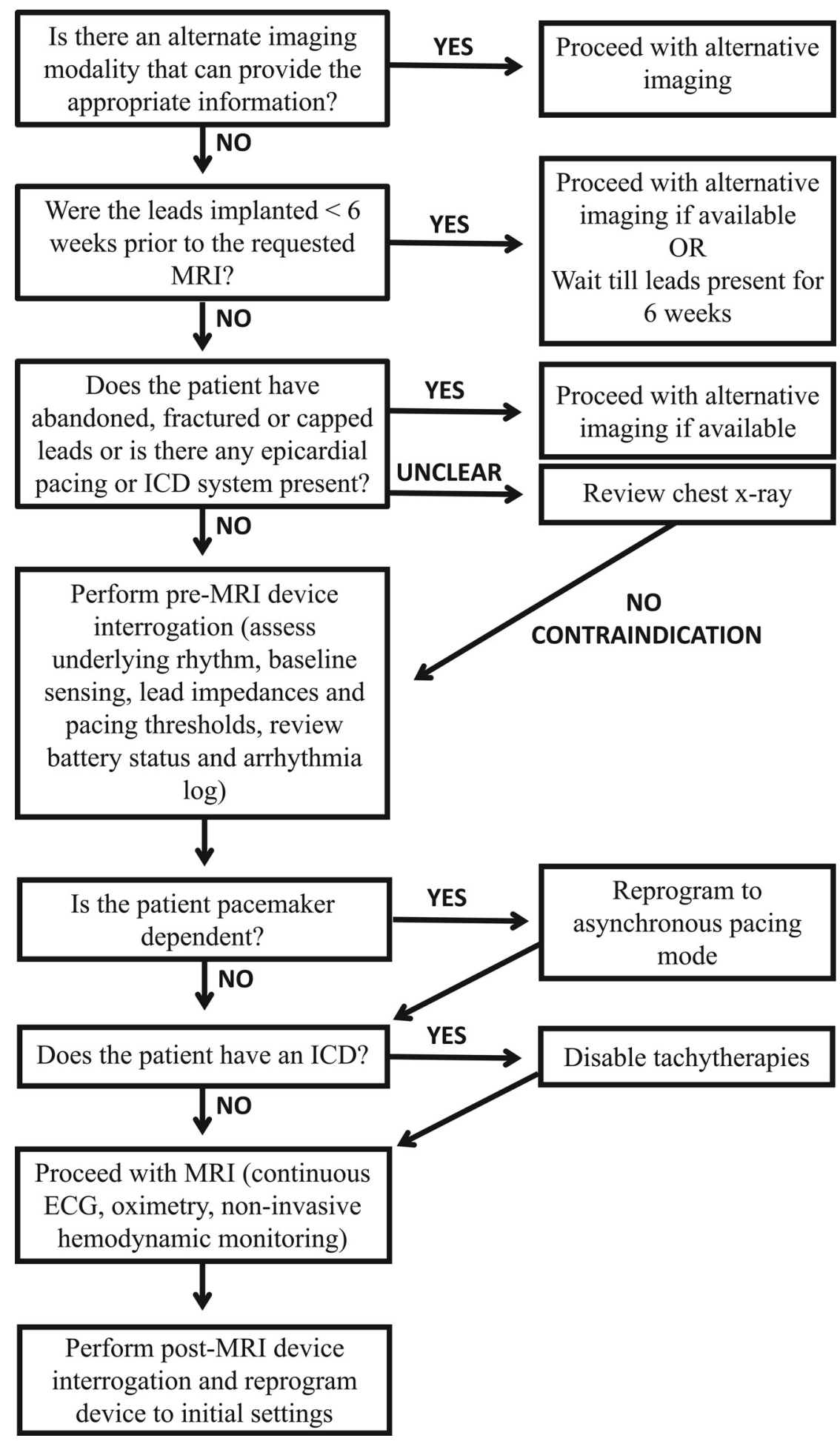

FIG 2. Comprehensive safety protocol: collaboration between neuroradiology and cardiology.

the body transmit coil because there is no body transmit/receive coil available. We believe that it would be contradictory and overly complicated to use a head transmit/receive coil followed by the body transmit coil and spine receive coil for imaging of the thoracic spine, which is the portion of the examination in which the CIED is positioned entirely within the coil.

In general, no alterations are made to our MR imaging protocols when a patient with a non-MR imaging-conditional CIED undergoes an MR imaging examination of the neural axis. In fact, any neurologic imaging study that can be run on a $1.5 \mathrm{~T}$ magnet, including sequences using echo-planar imaging, can be performed in a patient with a CIED, as long as the examination is performed in normal operating mode. This includes diffusion-weighted imaging, perfusion imaging, and diffusion tensor imaging. These factors are independent of the pacemaker device. In contrast to artifacts encountered due to the device generator and leads when performing cardiac or breast MR imaging examinations, no alterations to our scanning protocols were necessary to combat artifacts when scanning the neural axis in patients with a CIED. However, the longer acquisition times used for some sequences when scanning in the normal operating mode (eg, due to longer scan TRs) make some sequences more susceptible to motion artifacts.

\section{Cardiac Evaluation}

A comprehensive protocol was established through the collaboration between the department of radiology and the division of cardiology (Fig 2) to ensure patient safety when performing MR imaging in patients with a CIED. The comprehensive safety protocol is similar to that initially described by Nazarian et al. ${ }^{38,39}$ Understanding the potential interactions between MR imaging and

for $>6$ weeks. Examinations are typically performed with receiveonly coils. Although we have used head transmit/receive coils in the past to minimize patient RF exposure, we are not currently using head transmit/receive coils because these coils do not allow the use of parallel imaging in patients with non-MR imagingconditional CIEDs. Not using parallel imaging would lengthen and thus may degrade the examination. Many patients are also undergoing concurrent MR imaging of the spine for which we use
CIED systems is imperative to the preimaging patient evaluation as well as the risk and benefit discussion. The main risk categorizations include the following: 1) ferromagnetic interaction, leading to induced electrical currents, arrhythmia induction, devicesensing malfunction, or electrical resets; 2) lead thermal conduction causing loss of capture ("capture" refers to the excitation of heart tissue by a pacing stimulus, assuming that the strength of stimulus is sufficient and tissue is not in a refractory 
period) due to local myocardial edema and scar formation with subsequent increased pacing thresholds; 3 ) change in the pacing mode to asynchronous or inhibition of pacing; 4) inappropriate arrhythmia sensing, which may lead to ICD therapy or antitachycardia therapy (Fig 3); and 5) saturation of device storage for diagnostic and event data. ${ }^{23}$

All patients scheduled for an MR imaging examination are screened by the department of radiology at the time of scheduling for the presence of a CIED. If a non-MR imaging-conditional CIED is present, the patient's case is reviewed by a radiologist and alternative imaging is recommended when the radiologist or ordering physician determines that the clinical information needed could be obtained from a non-MR imaging technique. In patients for whom MR imaging is judged to be the most appropriate test, the radiologist makes this documentation in the patient's electronic medical record. Before imaging, the patient is then evaluated by an electrophysiologist (EP) in the same way that patients scheduled to undergo an operation are evaluated preoperatively. If scheduled as an outpatient, a patient with a non-MR imagingconditional CIED is referred to the cardiac EP clinic, where a physician performs a history, physical, electrocardiogram, and device interrogation. A chest radiograph is performed when there is uncertainty as to the presence of a fractured or retained transvenous pacing lead or a permanent epicardial pacing lead, each of which is an absolute contraindication to undergoing an MR imaging examination (Fig 4). Please refer to Table 3 for a complete list of absolute contraindications for undergoing MR imaging in patients with nonconditional CIEDs. If the patient has no absolute contraindication to MR imaging, the cardiologist discusses the potential risks and benefits with the patient, and the study is scheduled. If scheduled as an inpatient, a patient with a non-MR imaging-conditional CIED is evaluated by the EP consult service in a fashion similar to that in an outpatient. An important point of clarification is for patients who are not awake and alert for the MR imaging examination, such as those who are intubated or heavily sedated. These patients are unable to report pain or discomfort during the examination and are only imaged in circumstances in which the benefits of the procedure greatly outweigh the risk of a complication, which could potentially go unnoticed.

On the day of the examination, a staff radiologist obtains informed consent from the patient following a discussion of the risks and benefits of the procedure. A physician or appropriately credentialed EP nurse then performs a complete device interrogation to determine the underlying rhythm and measures baseline sensing, lead impedances, and pacing thresholds. All devices are programmed to an asynchronous pacing mode (DOO/VOO) (Table 1) during imaging in patients who are pacemaker-dependent; this mode is defined as the absence of a spontaneous underlying rhythm of $>40$ beats per minute. In those who are not pacemaker-dependent, devices may be placed in a mode in which backup pacing is possible (AAI/ VVI/DDI) or they may be placed in a mode in which pacing is disabled entirely (OVO/ODO). All ICD therapies to treat ventricular tachycardia or ventricular fibrillation are disabled before MR imaging.

Continuous electrocardiogram, pulse oximetry, and noninvasive blood pressure monitoring are performed, in addition to patient symptom assessment throughout imaging. Imaging was per-

FIG 3. Risk categorization: inappropriate device sensing during MR imaging. Intracardiac elect grams from a patient with a dual-chamber ICD. Electromagnetic interference is seen on both atrial and ventricular channels (solid arrows), resulting in oversensing (dashed arrow) and an inappropriate ICD shock (asterisk).
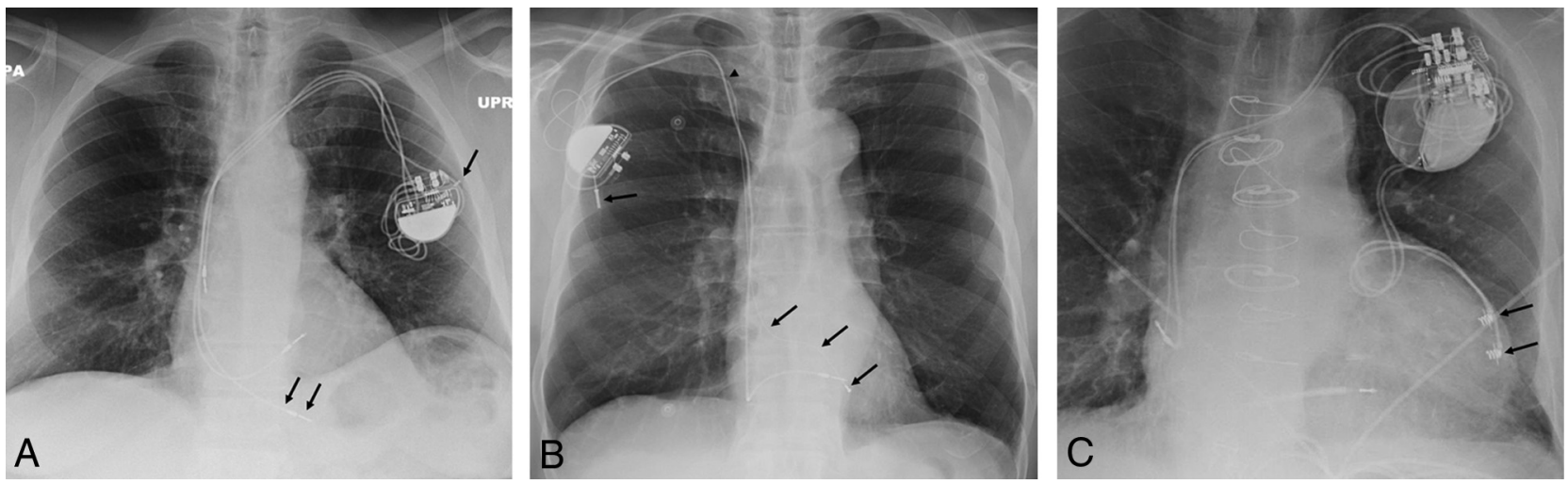

FIG 4. Absolute contraindications: chest $x$-ray examinations with abandoned and epicardial leads. Posteroanterior view of the chest $(A)$ demonstrates an abandoned lead (black arrows) in a patient with a dual-chamber pacemaker device. $B$, An abandoned right ventricular lead (black arrows) in a patient with a single-lead pacemaker device. The black arrowhead denotes a fracture of the abandoned lead. C, A patient with a biventricular ICD pacing system, which includes transvenous atrial and right ventricular leads and 2 permanent epicardial pacing leads (black arrows). None of these 3 patients would be cleared to undergo MR imaging examination at our institution. 
Table 3: Contraindications for undergoing MRI with a non-MRIconditional CIED

Contraindications

A device implanted $<6$ weeks prior to the MRI examination
Abandoned or fractured permanent intracardiac pacing leads
Permanent epicardial pacing leads
Temporary epicardial pacing leads when the device generator is
attached
Temporary intracardiac pacing leads (with or without attached
generator)
Intubated, obtunded, or heavily sedated patients (relative
contraindication)

formed under the continuous supervision of a member of the EP consult team until 2011, when it was determined that the risks in patients who were not pacemaker-dependent were low. Since that time, only patients who are pacemaker-dependent require continuous supervision by a member of the EP consult team during imaging. Patients who are not pacemaker-dependent are currently supervised by a radiology MR imaging technician and a radiology nurse, neither of whom routinely undergo additional training for this procedure. Resuscitation equipment and an external defibrillator with the capability of delivering transcutaneous pacing are immediately available. Imaging is terminated for any adverse events or if the safety of the patient is thought to be compromised.

On completion of the examination, a physician or appropriately credentialed EP nurse performs a repeat device interrogation with measurements, noting any changes in device settings, arrhythmias, therapies delivered, or battery depletion. Device settings are reprogrammed to the initial settings if any adjustments have been made previously or modified on the basis of postimaging observations. Patients are then followed routinely in their outpatient cardiac device clinics.

\section{Our Experience}

Using the electronic medical record of the hospital, we retrospectively identified $292 \mathrm{MR}$ imaging examinations of the neural axis that were performed in 121 patients with non-MR imaging-conditional pacemakers between June 2010 and November 2016. All examinations were performed at $1.5 \mathrm{~T}$ following appropriate cardiac clearance per the multidisciplinary protocol outlined in the "Cardiac Evaluation" section. Of the 292 MR imaging examinations, there were 162 MRIs of the brain (55\%), 26 MRAs of the head (9\%), 16 MRAs of the neck (5\%), 28 MRIs of the cervical spine (10\%), 26 MRIs of the thoracic spine (9\%), 32 MRIs of the lumbar spine (11\%), 1 MRI of the face/neck, and 1 MRI of the orbits. In patients requiring $>1 \mathrm{MR}$ imaging examination, multiple studies were grouped into single patient encounters when possible. In total, 204 discrete MR imaging encounters occurred in this group of 121 patients.

Seven of 121 patients ( $6 \%$ of patients with a total of $17 \mathrm{MR}$ imaging encounters) were pacemaker-dependent, 111 of 121 patients (92\%) were non-pacemaker-dependent, and in 3 of 121 patients (2\%), it was unclear whether the patient was pacemakerdependent or not. As described in the "Cardiac Evaluation" section, pacemakers were set to an asynchronous pacing mode in patients who were pacemaker-dependent.

All MR imaging examinations were completed safely with no clinically important complications reported. No examinations were terminated prematurely due to pacemaker-related problems. There were 8 episodes in 204 total encounters (4\%), in which minor, unexpected programming changes occurred with no immediate or delayed adverse outcomes. In 2 patients, MR imaging electromagnetic interference artifacts were noted in the ventricular fibrillation zone; however, no therapy was administered (as noted in the "Cardiac Evaluation" section, all ICD therapies to treat ventricular tachycardia or ventricular fibrillation are disabled before MR imaging). In these 2 patients, no subsequent change in device settings or postprocedural complication was noted following the MR imaging examination. In 1 patient with a dual-chamber pacemaker, there was a minor change in the right ventricular lead impedance, though the impedance remained within normal limits. In another patient with a dual-chamber pacemaker, a slight increase in the right atrial lead capture threshold was managed by a slight increase in the right atrial lead pacing output amplitude.

Variations in lead impedance and pacing capture thresholds have been noted previously but are generally deemed to be not clinically important and occur, to some degree, in the general CIED population. ${ }^{40,41}$ There were noise reversion episodes in 3 patients (multiple episodes in 2 of those patients). In all 3 patients, there were no immediate adverse outcomes, and the device was reprogrammed to the original settings after the MR imaging examination. In 1 patient with a single-lead pacemaker who experienced $6 \mathrm{MR}$ imaging encounters, the pacemaker mode converted to asynchronous pacing with a rate of 100 beats per minute during 1 of the $6 \mathrm{MR}$ imaging examinations. This patient had atrial fibrillation with an underlying ventricular rate ranging from the 40 s to the 50 s and required pacing $88 \%$ of the time. No subsequent adverse outcomes were reported in this patient. No programming changes or other unexpected events were recorded during the patient's other 5 encounters.

Our cohort of patients who were safely scanned with MR imaging included those who were pacer-dependent and others who underwent examinations of the thorax. Limitations of this study include the relatively low number of pacer-dependent patients (17/292 confirmed MR imaging encounters) and the general heterogeneity of specific CIED manufacturer generator/lead combinations. In addition, no pediatric patients were scanned at our institution, and no patients were scanned at field strengths of $>1.5 \mathrm{~T}$.

\section{CONCLUSIONS}

On the basis of our experience during the past 6 years performing MR imaging examinations in patients with non-MR imagingconditional CIEDs, including those who are pacemaker dependent or are undergoing an MR imaging of the thorax, as well as the growing body of supportive evidence in the literature, it is our opinion that these examinations can be performed safely when risks to the patient are well-managed. Before proceeding with an MR imaging examination, it is important to confirm that doing so is judged appropriate following a thorough review of the patient's chart and a discussion with the ordering physician by the supervising radiologist. Strict adherence to basic screening and scan protocols is also required. Patient populations that should con- 
tinue to be excluded from MR imaging include those with devices implanted $<6$ weeks prior, those with retained or fractured device leads, those with surgically placed permanent epicardial pacing leads, and those with temporary intracardiac/epicardial pacer devices with the external generator still attached.

\section{ACKNOWLEDGMENTS}

The authors thank Charles Fasanati for his help in preparing this article.

Disclosures: Jeremy D. Collins-UNRELATED: Consultancy: Guerbet, Comments: advisory board meeting; Grants/Grants Pending: Siemens, Comments: Conebeam CT grant for hepatocellular carcinoma. Bradley P. Knight-UNRELATED: Grant: Medtronic, Boston Scientific, St. Jude Medical, BIOTRONIK*; Consulting Fee or Honorarium: Medtronic, Boston Scientific, St. Jude Medical, BIOTRONIK. Alexander J. Nemeth-UNRELATED: Grants/Grants Pending: National Institutes of Health, Comments: RO1HL092259, SP0039078, coinvestigator 5\% effort salary support for Cerebral Autoregulation Monitoring to Reduce Brain Injury from Cardiac Surgery. *Money paid to the institution.

\section{REFERENCES}

1. OECD. Magnetic resonance imaging (MRI) exams, total. Health: Key Tables. OECD 2014. https://data.oecd.org/healthcare/magneticresonance-imaging-mri-exams.htm. Accessed December 24, 2016

2. Nazarian S, Hansford R, Roguin A, et al. A prospective evaluation of a protocol for magnetic resonance imaging of patients with implanted cardiac devices. Ann Intern Med 2011;155:415-24 CrossRef Medline

3. Kanal E, Barkovich AJ, Bell C, et al; ACR Blue Ribbon Panel on MR Safety. ACR guidance document for safe MR practices: 2007. AJR Am J Roentgenol 2007;188:1447-74 CrossRef Medline

4. Levine GN, Gomes AS, Arai AE, et al; American Heart Association Committee on Diagnostic and Interventional Cardiac Catheterization, American Heart Association Council on Clinical Cardiology, American Heart Association Council on Cardiovascular Radiology and Intervention. Safety of magnetic resonance imaging in patients with cardiovascular devices: an American Heart Association scientific statement from the Committee on Diagnostic and Interventional Cardiac Catheterization, Council on Clinical Cardiology, and the Council on Cardiovascular Radiology and Intervention: endorsed by the American College of Cardiology Foundation, the North American Society for Cardiac Imaging, and the Society for Cardiovascular Magnetic Resonance. Circulation 2007;116:2878-91 CrossRef Medline

5. Roguin A, Schwitter J, Vahlhaus C, et al. Magnetic resonance imaging in individuals with cardiovascular implantable electronic devices. Europace 2008;10:336-46 CrossRef Medline

6. Wilkoff BL, Bello D, Taborsky M, et al; EnRhythm MRI SureScan Pacing System Study Investigators. Magnetic resonance imaging in patients with a pacemaker system designed for the magnetic resonance environment. Heart Rhythm 2011;8:65-73 CrossRef Medline

7. Sommer T, Naehle CP, Yang A, et al. Strategy for safe performance of extrathoracic magnetic resonance imaging at 1.5 Tesla in the presence of cardiac pacemakers in non-pacemaker-dependent patients: a prospective study with 115 examinations. Circulation 2006;114: 1285-92 CrossRef Medline

8. Russo RJ, Costa HS, Silva PD, et al. Assessing the risks associated with MRI in patients with a pacemaker or defibrillator. $N$ Engl J Med 2017;376:755-64 CrossRef Medline

9. Martin ET, Coman JA, Shellock FG, et al. Magnetic resonance imaging and cardiac pacemaker safety at 1.5-Tesla. J Am Coll Cardiol 2004;43:1315-24 CrossRef Medline

10. Dandamudi S, Collins JD, Carr JC, et al. The safety of cardiac and thoracic magnetic resonance imaging in patients with cardiac implantable electronic devices. Acad Radiol 2016;23:1498-505 CrossRef Medline

11. Aguilera AL, Volokhina YV, Fisher KL. Radiography of cardiac con- duction devices: a comprehensive review. Radiographics 2011;31: 1669-82 CrossRef Medline

12. Hunter TB, Taljanovic MS, Tsau PH, et al. Medical devices of the chest. Radiographics 2004;24:1725-46 CrossRef Medline

13. Kirk M. Basic principles of pacing. In: Chow AW, Buxton AE, eds. Implantable Cardiac Pacemakers and Defibrillators: All You Wanted to Know. Oxford, UK: Blackwell Publishing; 2006:1-28 CrossRef

14. Kalin R, Stanton MS. Current clinical issues for MRI scanning of pacemaker and defibrillator patients. Pacing Clin Electrophysiol 2005;28:326-28 CrossRef Medline

15. Langman DA, Goldberg IB, Finn JP, et al. Pacemaker lead tip heating in abandoned and pacemaker-attached leads at 1.5 Tesla MRI. J Magn Reson Imaging 2011;33:426-31 CrossRef Medline

16. Pavlicek W, Geisinger M, Castle L, et al. The effects of nuclear magnetic resonance on patients with cardiac pacemakers. Radiology 1983;147:149-53 CrossRef Medline

17. Kanal E, Shellock FG, Talagala L. Safety considerations in MR imaging. Radiology 1990;176:593-606 CrossRef Medline

18. Horwood L, Attili A, Luba F, et al. Magnetic resonance imaging in patients with cardiac implanted electronic devices: focus on contraindications to magnetic resonance imaging protocols. Europace 2017;19:812-17 CrossRef Medline

19. Naehle CP, Strach K, Thomas D, et al. Magnetic resonance imaging at 1.5-T in patients with implantable cardioverter-defibrillators. J Am Coll Cardiol 2009;54:549-55 CrossRef Medline

20. Higgins JV, Sheldon SH, Watson RE Jr, et al. "Power-on resets" in cardiac implantable electronic devices during magnetic resonance imaging. Heart Rhythm 2015;12:540-44 CrossRef Medline

21. Ferreira AM, Costa F, Tralhao A, et al. MRI-conditional pacemakers: current perspectives. Med Devices (Auckl) 2014;7:115-24 CrossRef Medline

22. Luechinger R, Duru F, Zeijlemaker VA, et al. Pacemaker reed switch behavior in $0.5,1.5$, and 3.0 Tesla magnetic resonance imaging units: are reed switches always closed in strong magnetic fields? Pacing Clin Electrophysiol 2002;25:1419-23 CrossRef Medline

23. van der Graaf AW, Bhagirath P, Götte MJ. MRI and cardiac implantable electronic devices; current status and required safety conditions. Neth Heart J 2014;22:269-76 CrossRef Medline

24. Ahmed FZ, Morris GM, Allen S, et al. Not all pacemakers are created equal: MRI conditional pacemaker and lead technology. J Cardiovasc Electrophysiol 2013;24:1059-65 CrossRef Medline

25. Boutet C, Mansourati J, Ben Salem D. How to manage central nervous system MRI with a cardiac implantable electronic device? J Neuroradiol 2016;43:307-08 CrossRef Medline

26. Luechinger R, Zeijlemaker VA, Pedersen EM, et al. In vivo heating of pacemaker leads during magnetic resonance imaging. Eur Heart J 2005;26:376-83; discussion 325-27 CrossRef Medline

27. Mollerus M, Albin G, Lipinski M, et al. Cardiac biomarkers in patients with permanent pacemakers and implantable cardioverterdefibrillators undergoing an MRI scan. Pacing Clin Electrophysiol 2008;31:1241-45 CrossRef Medline

28. Sommer T, Naehle CP, Schild H. Magnetic resonance imaging in patients with cardiac pacemakers. J Am Coll Cardiol 2005;46:56162; author reply 562 Medline

29. Collins CM, Wang Z. Calculation of radiofrequency electromagnetic fields and their effects in MRI of human subjects. Magn Reson Med 2011;65:1470-82 CrossRef Medline

30. Baker KB, Tkach JA, Nyenhuis JA, et al. Evaluation of specific absorption rate as a dosimeter of MRI-related implant heating. J Magn Reson Imaging 2004;20:315-20 CrossRef Medline

31. International Electrotechnical Commision. Medical electrical equipment - Part 2-33: Particular requirements for the basic safety and essential performance of magnetic resonance equipment for medical diagnosis. IEC 60601-2-33 2010

32. Calcagnini G, Triventi $M$, Censi $F$, et al. In vitro investigation of pacemaker lead heating induced by magnetic resonance imaging: role of implant geometry. J Magn Reson Imaging 2008;28:879-86 CrossRef Medline 
33. Nitz WR, Oppelt A, Renz W, et al. On the heating of linear conductive structures as guide wires and catheters in interventional MRI. $J$ Magn Reson Imaging 2001;13:105-14 Medline

34. Nordbeck P, Ritter O, Weiss I, et al. Impact of imaging landmark on the risk of MRI-related heating near implanted medical devices like cardiac pacemaker leads. Magn Reson Med 2011;65:44-50 CrossRef Medline

35. Mattei E, Triventi M, Calcagnini G, et al. Complexity of MRI induced heating on metallic leads: experimental measurements of 374 configurations. Biomed Eng Online 2008;7:11 CrossRef Medline

36. Nordbeck P, Fidler F, Weiss I, et al. Spatial distribution of RF-induced E-fields and implant heating in MRI. Magn Reson Med 2008; 60:312-19 CrossRef Medline

37. Nordbeck P, Weiss I, Ehses P, et al. Measuring RF-induced currents inside implants: impact of device configuration on MRI safety of cardiac pacemaker leads. Magn Reson Med 2009;61:570-78 CrossRef Medline
38. Nazarian S, Halperin HR. How to perform magnetic resonance imaging on patients with implantable cardiac arrhythmia devices. Heart Rhythm 2009;6:138-43 CrossRef Medline

39. Nazarian S, Roguin A, Zviman MM, et al. Clinical utility and safety of a protocol for noncardiac and cardiac magnetic resonance imaging of patients with permanent pacemakers and implantable-cardioverter defibrillators at 1.5 Tesla. Circulation 2006;114:1277-84 CrossRef Medline

40. Gimbel JR, Bello D, Schmitt M, et al; Advisa MRI System Study Investigators. Randomized trial of pacemaker and lead system for safe scanning at 1.5 Tesla. Heart Rhythm 2013;10:685-91 CrossRef Medline

41. Mollerus M, Albin G, Lipinski M, et al. Magnetic resonance imaging of pacemakers and implantable cardioverter-defibrillators without specific absorption rate restrictions. Europace 2010;12:947-51 CrossRef Medline 